ARTÍCULO

\title{
El reconocimiento de las diferencias como fundamento para la educación inclusiva: la evaluación como barrera en el discurso docente
}

\author{
Jorge Eduardo Alfaro' (D) \\ Valeria Herrera Fernández" (D)
}

\section{RESUMEN}

En Chile un importante número de estudiantes provenientes de grupos minoritarios, se encuentran excluidos de participar en el sistema educativo dadas sus diferencias culturales, lingüísticas, biológicas o sociales. La teoría del reconocimiento propone un sistema de categorías que permite analizar las respuestas educativas formuladas para superar barreras de acceso, participación y progreso en la trayectoria escolar. Para ello, se propone analizar críticamente el discurso de los profesores, como principales actores involucrados en la implementación cotidiana de las políticas educativas sobre inclusión, en espacios educativos en los que se reproducen prácticas y experiencias arraigadas en una cultura escolar tradicional. Evidenciar estos discursos permitirá aproximarse a la comprensión del problema de participación y progreso académico en el sistema educacional de los estudiantes de grupos minoritarios.

PALABRAS CLAVE

educación inclusiva; reconocimiento; diversidad. 


\title{
THE RECOGNITION OF DIFFERENCES AS A BASIS FOR INCLUSIVE EDUCATION: ASSESSMENT AS BARRIER AT TEACHER SPEECH
}

\begin{abstract}
In Chile, a significant number of students from minority groups are excluded from participating in the educational system due to their cultural, linguistic, biological or social differences. The theory of recognition proposes a system of categories that allows analyzing the educational responses formulated to overcome barriers of access, participation and progress in the school trajectory. To do this, it is proposed to critically analyze the discourse of teachers, as the main actors involved in the daily implementation of educational policies on inclusion, in educational spaces in which practices and experiences rooted in a traditional school culture are reproduced. Evidencing these discourses will allow us to approach the understanding of the problem of participation and academic progress in the educational system of students from minority groups.
\end{abstract}

KEYWORDS

inclusive education; recognition; diversity.

\section{RECONHECIMENTO DAS DIFERENÇAS COMO BASE PARA A EDUCAÇÃO INCLUSIVA: A AVALIAÇÃO COMO BARREIRA NO DISCURSO DO PROFESSOR}

\section{RESUMO}

No Chile, um número significativo de estudantes de grupos minoritários é excluído da participação no sistema educacional, dadas suas diferenças culturais, linguísticas, biológicas ou sociais. A teoria do reconhecimento propõe um sistema de categorias que permite analisar as respostas educacionais formuladas para superar barreiras de acesso, participação e progresso na trajetória escolar. Para esse fim, propõe-se analisar criticamente o discurso dos professores como os principais envolvidos na implementação diária das políticas de educação para a inclusão, em espaços educacionais onde as práticas e experiências arraigadas são reproduzidas em uma cultura escolar tradicional. Provar esses discursos nos permitirá abordar a compreensão do problema da participação e do progresso acadêmico no sistema educacional dos estudantes das minorias.

PALAVRAS-CHAVE

educação inclusiva; reconhecimento; diversidade. 


\section{ANTECEDENTES}

Durante las últimas décadas, las políticas educativas promovidas a nivel global han generado nuevas acciones para garantizar el acceso y la promoción de aprendizajes de niños, niñas y jóvenes en los distintos Estados en todo el mundo (UNESCO, 2017). En este contexto, pareciera ser que la primera pregunta a responder es cómo lograr más y mejores aprendizajes para los y las estudiantes, más allá de toda diferencia o condición. Para aproximarnos a una eventual respuesta, resulta fundamental conocer a aquellos grupos que tradicionalmente han sido excluidos del sistema social dada su heterogeneidad, para saber cómo hacerlo, primero hay que saber quiénes son aquellos que necesitan aprender más y mejor. Si entendemos la educación como un bien material utilizado para progresar, la pregunta sobre cómo aprender o cómo enseñar, sitúa la discusión en torno a los bienes (Hobbes, 1651), en este caso, los educativos.

Al aplicar las categorías de Hobbes a la educación, esta estaría determinada por el acceso a objetivos, experiencias y el poder que pudiera alcanzarse producto de ella. Aceptar esta premisa, que en una sociedad capitalista podría en efecto tener sentido, implicaría asumir que los sujetos que participan de esta lucha se encuentran en un acuerdo equitativo, libre y en igualdad de condiciones (Habermas, 1991). Sin embargo desde una aproximación material, la educación es un campo en el que los individuos no se encuentran en posiciones equitativas para participar de un debate libre, toda vez que el poder es ejercido principalmente desde los grupos en posiciones privilegiadas hacia los minoritarios (desde los adultos hacia los niños y niñas, desde las mayorías lingüísticas y culturales hacia las minorías, desde los grupos de poder socioeconómico hacia los más pobres, desde lo urbano hacia lo rural, etcétera) como forma de dominación y colonización (Santos, 2013).

Enfocar la problemática educativa desde los bienes, niega el carácter desigual de las relaciones educativas y por tanto deja de considerar activamente a quienes se encuentran en una posición jerárquica inferior. En ese sentido, es necesario recurrir a otra categoría que permita explicar la dificultad que existe para promover acceso a más y mejores aprendizajes. Si la lucha actual por bienes materiales es jerárquica, el supuesto que subyace es que una parte de los individuos no logra progresar en tanto no encuentran actos lingüístico-comunicativos propios y libres de coerción para participar del debate (Honneth, 2009). Por ello el reconocimiento entre individuos, para poder participar del debate, constituiría un elemento que antecede la lucha por los bienes, materiales y educativos.

El Reconocimiento es una categoría que al incorporar el carácter intersubjetivo de las relaciones, modifica la teoría de Hobbes respecto de la lucha del "todos contra todos", afirmando que las injusticias sociales no solo están determinadas por aspectos materiales, sino en primer término por la falta de reconocimiento (Honneth, 1992). La subjetividad, la cultural, la lengua, la identidad, entre otros rasgos constituyen elementos necesarios a ser reconocidos para poder participar en igualdad de condiciones, de lo contrario la relación se expresa en términos del abuso de poder y desigualdad (Van Dijk, 2009). En este sentido, las injusticias no solo se resuelven redistribuyendo recursos sino además reconociendo a los sujetos como interlocutores válidos al momento de evidenciarse la injusticia (Fraser y Honneth, 
2003) toda vez que, para participar de un debate, es necesario los participantes se reconozcan entre ellos en igualdad de condiciones para proponer juicios racionales a fin de tomar determinaciones (Honneth, 2014).

Según Honneth (1992) el reconocimiento se constituye por tres esferas: la esfera emocional vinculada a las relaciones de amor, en la cual las personas encuentran satisfecha la necesidad de afecto, la esfera normativa en la que las personas se reconocen a sí mismas en relaciones jurídicas en que el otro es entendido como un sujeto contractual y de derecho, para finalmente alcanzar la esfera ética, en la que se alcanza la abstracción de cualquier condicionante o característica que pudiera impedir la consideración del otro como persona en igualdad de condiciones. La falta de reconocimiento en cualquiera de esas tres esferas ha sido denominada por Honneth como desprecio o menosprecio.

En el plano educativo el concepto de educación inclusiva, contribuye a tensionar con mayor fuerza la necesidad de reflexionar respecto de cómo la educación, se entiende desde la lucha del reconocimiento y no desde la lucha por los bienes. En este contexto, es importante definir la educación inclusiva como una filosofía educativa que se cuestiona problemáticas agobiantes relacionadas con la injusticia y la desigualdad humanas, así como también se ocupa de desnaturalizar prácticas discriminatorias con miras a la conformación y transformación para un mundo mejor (Echeita y Navarro, 2015). Para alcanzarlo, se ha configurado una triada entre cultura, políticas y prácticas inclusivas (Booth y Ainscow, 2015) que sustentadas en valores y en una forma de compresión de la diversidad, habilita a los centros educativos a realizar los ajustes necesarios para garantizar accesibilidad y universalidad en el aprendizaje (Boggino y Boggino, 2013). Desde este entendimiento, la educación inclusiva cuestiona que determinados individuos o colectivos, permanezcan excluidos del debate educacional, y por tanto, no reciban una respuesta ajustada a sus características y necesidades.

La necesidad de problematizar sobre educación inclusiva radica en que, siendo un derecho humano, esto no ha sido condición suficiente para eliminar toda forma de discriminación, segregación y/o exclusión. El último informe de Educación para Todos (UNESCO, 2015) establece que el 50\% de los países no logra cumplir con el deber de proveer educación a la primera infancia, siendo los más pobres, las personas con discapacidad, las mujeres, las personas pertenecientes a minorías étnicas, las personas con VIH/SIDA, las personas de sectores rurales y los niños, niñas y jóvenes trabajadores los más afectados por la exclusión social.

En Chile son las desigualdades socioeconómicas, geográficas, de género, de capacidad y de edad las que explican las principales desigualdades (OECD, 2017; ONU, 2019; SENADIS, 2015). El factor común de dichas desigualdades parece estar relacionado con la pertenencia a distintos grupos de personas que tradicionalmente han sido excluidos o segregados del sistema educativo.

$\mathrm{Al}$ respecto, la investigación internacional ha profundizado en los bienes materiales e inmateriales que obstaculizan el ejercicio del derecho a la educación. En este contexto se advierte que las principales barreras para ejercer este derecho, se encuentran en las concepciones desactualizadas sobre educación y diversidad existentes en las comunidades educativas y sociedad en general, la falta de trabajo co- 
laborativo, la necesidad de formación, los recursos pedagógicos y el rol de los líderes educativos (Alborno y Gaad, 2014; Bernabé, Alonso y Bermell, 2016; Engelbrecht et al., 2017; López et al., 2014; Mackey, 2014; Mellado et al., 2017). Estos factores han permitido visibilizar de qué modo la distribución de recursos, ya sean materiales (infraestructura, material pedagógico, recursos económicos, etc.) o simbólicos (conocimientos, liderazgos, estatus epistémico, etc.), obstaculizan el desarrollo de competencias para la educación inclusiva. Sin embargo, no se han abordado las experiencias de reconocimiento y menosprecio que les subyacen (Salas, 2017) y que en alguna medida, podrían explicar por qué no se ha priorizado esta distribución.

Durante la última década Chile ha avanzado en esta materia, promulgando leyes y decretos para evitar la exclusión y la segregación, y promover la no discriminación. Entre las más significativas podemos mencionar la ley n. 20.422/2010 sobre Igualdad de Oportunidades e Inclusión Social de personas con Discapacidad, la ley n. 20.609/2012 que establece medidas contra la discriminación y la ley n. 20.845/2015 que regula la admisión, elimina el financiamiento compartido y prohíbe el lucro en la educación. Junto con ello, existe el decreto n. 170/2009 que fija las normas para determinar los alumnos que presentan necesidades educativas especiales (NEE) y que serán eventuales beneficiarios de las subvenciones para educación especial y el decreto n. 83/2015 que aprueba criterios y orientaciones de adecuación curricular para estudiantes con NEE de educación parvularia y básica. En la mayoría de los centros educativos en Chile, las propuestas de inclusión se articulan en Programas de Integración Escolar (PIE) que otorgan apoyos especializados a estudiantes diagnosticados con NEE.

En general, este tipo de normativas se centra en garantizar el acceso a la educación (Booth y Ainscow, 2015), no alcanzando a promover la participación y el progreso académico de los estudiantes. Asimismo, este cuerpo legal aborda la educación inclusiva principalmente desde aspectos económicos o médico-clínicos (Peña, 2013). De esta manera, la estrategia diseñada centra sus esfuerzos en determinar problemáticas en los y las estudiantes a través de una evaluación sustentada sobre el valor de la igualdad (Dubet, 2014; Stobart, 2008). A través de una evaluación normalizadora se tiende a comparar al grupo minoritario con el grupo mayoritario con la expectativa de homogenizar al grupo en general. De esta forma, se proponen acciones educativas que atienden a características de determinados grupos, más que a promover un sistema educativo capaz de responder a las diversas formas con que enfrentan las barreras de participación y aprendizaje presentes en dicho sistema. En este contexto, la investigación se aproxima al fenómeno de la educación inclusiva, a través del análisis crítico del discurso docente sobre inclusión y sobre la consideración de experiencias de reconocimiento y menosprecio en los espacios educativos.

\section{METODOLOGÍA}

El objetivo del presente trabajo es analizar críticamente el discurso de los profesores, como principales actores involucrados en la implementación cotidiana de las políticas educativas sobre inclusión. La investigación se abordó desde un paradigma sociocrítico con enfoque cualitativo asumiendo que la pretensión del 
conocimiento es formular una transformación del fenómeno y la sociedad (Villalobos, 2010). El estudio se realizó en dos establecimientos públicos de educación básica de la comuna de Puerto Montt, en el sur de Chile. El primero es una escuela de buen rendimiento académico de acuerdo con la evaluación del actual Sistema de Medición de la Calidad de la Educación (SIMCE), mientras el segundo se encuentra bajo la media comunal, de acuerdo a la misma medición. El primer establecimiento se reconoce como una escuela de excelencia, mientras el segundo es un establecimiento que surge como una oferta educativa para estudiantes con bajo rendimiento. Se entrevistó a 30 profesores ( 27 mujeres y 3 hombres) con una edad promedio de 38 años, 41 horas de contrato semanal, 14 años de experiencia laboral y ocho años de ejercicio profesional en el establecimiento. Los docentes abarcan todas las áreas disciplinares del currículo nacional, en los niveles de educación parvularia, básica y la modalidad de educación especial. Todos recibieron información con los objetivos y alcances de la investigación, junto a un consentimiento informado autorizando su participación en las entrevistas.

Las entrevistas realizadas se estudiaron en base a un análisis crítico del discurso, toda vez que esta herramienta permite profundizar en las estructuras gramaticales y semánticas de los entrevistados evitando las resistencias propias y los discursos políticamente correctos relacionados con esta temática (Cameron y Panovic, 2014). En este sentido, los estudios críticos del discurso, entendidos como una práctica en la que se habla sobre algo, constituyen "un movimiento intelectual específicamente interesado en la elaboración de la teoría y el análisis crítico de la reproducción discursiva del abuso de poder y de la desigualdad social" (Van Dijk, 2009, p. 19). La investigación sobre las formas de reconocimiento y menosprecio en la interacción humana, encuentra un soporte metodológico en el análisis crítico del discurso que permite visibilizar aquello que la descripción no devela.

Para alcanzar el objetivo de estudio se optó por el modelo tridimensional de Fairclough (2008), que permite analizar el discurso primero como texto, luego como práctica y finalmente como práctica social. Inicialmente se identifican en el corpus las distintas estrategias discursivas que utilizan los hablantes para justificar sus enunciados (vocabulario, gramática, cohesión, estructura, actos de habla). Posteriormente se describen relaciones de poder y otras patologías sociales que existen en el discurso para finalmente situarlas en el contexto político y social en que se configuran y operan.

\section{RESULTADOS}

Este apartado presenta los análisis realizados al discurso docente respecto del currículo, la evaluación y la tensión entre homogeneización y diversificación en el sistema educativo. En este sentido, se discute la idea de que la implementación del currículo permite disponer de experiencias de menosprecio hacia las diferencias de los estudiantes desde sus sistemas de evaluación homogeneizantes sustentados en el valor de la igualdad. Se observa que los profesores ocupan una serie de estrategias discursivas para justificar concepciones y prácticas excluyentes y falta de formación 
y inseguridades respecto de la diversidad. Al respecto, se presenta el discurso de una profesora de educación básica refiriendo a su experiencia en la implementación de políticas de educación inclusiva.

Cita 1 - Profesora 2: "Nosotros tenemos que ocupar las horas de clases para enseñar, a la hora de la evaluación tenemos que ver el resultado si eso dio resultado o no ¿Cómo yo me voy a fiar de que me dio resultado si en la hora de la prueba estamos haciéndole, leyéndole todo, haciéndole todo a la niña? Entonces yo quiero saber si mis estrategias resultan para que al momento de aplicar la evaluación las niñas sean autónomas".

La profesora de la cita 1 utiliza la evaluación para justificar la homogeneidad de su práctica pedagógica. Comienza utilizando al verbo deóntico "tenemos que", para situar una obligación sin agente activo, en este sentido no explicita quién la obliga a ocupar las horas de clases para enseñar, ni quién la obliga a ver el resultado de ello, quedando la responsabilidad de esta acción en el anonimato.

Habiendo establecido esta situación, luego la profesora va a diferenciar la enseñanza de la evaluación, esta última asociada al resultado de manera dicotómica (hay resultado o no hay resultado). Al hacer esta separación entre dos procesos educativos articulados (Hattie, 2017) la profesora justifica la necesidad de contar con prácticas homogéneas para todas sus estudiantes. $\mathrm{Al}$ respecto, utiliza una pregunta de manera retórica a través de un acto de habla interrogativo apelativo " ¿cómo yo me voy a fiar de que me dio resultado?", invalidando los apoyos que se le prestan a la estudiante para mostrar sus aprendizajes. Continúa utilizando el hipérbaton a modo retórico “leyéndole todo, haciéndole todo", lo que la faculta para describir como límites para el logro de la autonomía, los apoyos diversificados que una o más estudiantes pudieran necesitar. El uso posterior de la partícula cohesiva "entonces" le permite justificar que, con el foco en los resultados, la evaluación separada del proceso de enseñanza, debe dar cuenta de los resultados y de las estrategias que los originaron, más que del proceso de aprendizaje cursado por las estudiantes.

En este contexto, la diversificación de la enseñanza (decreto n. 83/2015) se presenta como un proceso indeseado que no arroja resultados. En el nivel de la práctica social se observa que al entender la evaluación como un mecanismo de control de la enseñanza, esta tiende a la homogeneización (Stobart, 2008). Lo que parece interesar al momento de evaluar no es qué aprenden ni cómo aprenden los diversos estudiantes (Hattie, 2017), sino cómo son los resultados de la enseñanza diseñada. Desde una concepción tradicional de enseñanza, la evaluación necesita dar cuenta de resultados y por tanto se homogeneiza. El valor que subyace a esta premisa es la igualdad (Dubet, 2014), expectativa de que los y las estudiantes sean lo más similares posibles y por tanto puedan recibir lo mismo. Bajo este argumento se justifica la negación de la diversificación de los procesos educativos y se establece la base para justificar la segregación, en tanto al pretenderse la igualdad, habría que agrupar a aquellos estudiantes similares entre sí.

La cita 2 refuerza la idea anterior, en la que otra profesora describe estrategias utilizadas para responder a la diversidad de estudiantes en el aula. 
Cita 2 - Profesora 15: "O sea se sabe que, que esas niñas requieren un poco más de apoyo pero las compañeras no las miran como que no pueden, eh no pueden realizar determinada actividad porque son niñas del PIE, no para nada, de hecho a ellas, por lo menos yo, en mi asignatura, las evalúo igual que a las demás compañeras, a no ser que vea que tengan un bajísimo, no es cierto, y veamos que tengan muchas dificultades, se hace alguna adecuación".

La profesora conforma dos grupos de estudiantes identificando a uno de ellos como "esas niñas" y al otro como "las demás compañeras". El demostrativo "esas" marca distancia respecto del receptor del mensaje, en contraposición con el sustantivo colectivo "compañeras" que está dotado de una carga semántica identitaria y comunitaria. Se podría inferir que "las demás compañeras" son parte del sistema educativo, mientras que "esas niñas" son ajenas. La profesora niega de manera explícita una expectativa que "esas niña [...] no pueden realizar determinada actividad". En este sentido, la estructura organizativa de su discurso deja entrever que podría existir una expectativa entre los profesores respecto de la capacidad de "esas niñas" para realizar o no determinadas actividades, argumentado que forman parte de un grupo minoritario, "porque son niñas del PIE". Sin embargo, la profesora obviará las diferencias entre las estudiantes, justificando su discurso a través el valor de la igualdad: "las evalúo igual que a las demás compañeras". La lógica discursiva radica en que se cree que las estudiantes no pueden hacer algo por pertenecer a un grupo minoritario, se contra argumenta estableciendo que las estudiantes sí pueden hacer ese algo a pesar de ser parte del grupo minoritario que originó la creencia, como ellas pueden hacer ese algo, se les evalúa igual que a las demás. Una vez que la profesora demuestra que las estudiantes pueden hacer ese algo, elimina inmediatamente las diferencias propias para enfrentar el aprendizaje, imponiéndose una noción de asimilación educativa sustentada en una evaluación estandarizada que a su vez se funda en el valor de la igualdad.

Aun así, la profesora identifica las diferencias, motivo por el cual conforma los dos grupos de estudiantes. Esta conformación, le permite detectar dificultades en el aprendizaje que justifican la necesidad de ajustar su evaluación. En este sentido, el reconocimiento de las diferencias lejos de aparecer como un mecanismo para legitimar al otro y garantizar su participación, se presenta como una forma de menosprecio (Honneth, 2009) en la que el individuo es reconocido para ser primero patologizado y luego asimilado al grupo mayoritario.

En la cita, la profesora se refiere al nivel de los estudiantes, empleando el adjetivo "bajisimo" como una característica de este. Esta oración además de no concluirla, la mitiga utilizando la expresión "no es cierto". Además de dar cuenta de que dice algo socialmente aceptado, busca complicidad en el otro y aceptación para así afirmar su argumento.

Se infiere que el hacer ajustes a la evaluación por causa de dificultades de aprendizaje (propias de "esas estudiantes"), resulta un mecanismo poco deseado puesto que atenta contra la estandarización asociada al valor de igualdad que anteriormente se relevó como deseado.

En este sentido, la evaluación cobra un ideal de justicia en la cual se asocia más a la igualdad que a la proporcionalidad (Rawls, 1971). Entendida la evaluación 
desde la igualdad no resulta justo que los y las estudiantes estén expuestos a experiencias de evaluación diferentes, sobre todo cuando algunas de estas experiencias se reconocen como más sencillas que otras. Si se entendiera la evaluación desde la proporcionalidad, podría justificarse la diversificación puesto que se reconocería en cada estudiante la posibilidad de aprender (enseñanza) y demostrar lo aprendido (evaluación) de acuerdo con sus características.

Este entendimiento de una educación, un currículum y una evaluación desde los resultados educativos, sitúa las diferencias de los y las estudiantes como un obstaculizador, más que como una condición necesaria de cualquier proceso inclusivo (Booth y Ainscow, 2015). Diversificar la enseñanza aparece como un elemento indeseado frente al valor que se otorga a un sistema educativo homogeneizante basado en evaluaciones de resultados y prácticas pedagógicas estandarizadas.

En la cita 3, por el contrario, otra profesora describe su experiencia en contextos que promueven la implementación de las políticas de educación inclusiva. La profesora plantea sus inseguridades y las estrategias que utilizó para superarlas.

Cita 3 - Profesora 4: "Al comienzo, tenía la verdad yo en lo personal mucho temor, tenía mucho miedo yo dije, yo necesito que ellos también tengan un resultado, cómo lo vamos a medir, lo que es la evaluación me costó mucho evaluar, después me di cuenta que no, que ellos tenían sus propios niveles, un propio piso para ir aprendiendo y de ahí ya fui como adaptándome más que nada eh yo a ellos y ellos también conociéndome a mí y dándose cuenta que podíamos trabajar en conjunto".

En la cita se visualiza una primera forma de menosprecio frente a las diferencias de los y las estudiantes, basada en la emoción del miedo a causa de la falta de resultados académicos. Al igual que en la cita anterior, la profesora conforma dos grupos de estudiantes a través de la expresión "ellos también", la cual da a entender que hay un grupo de estudiantes que obtiene resultados y un grupo que no los obtiene, y debería. El miedo se presenta cuando la enseñanza que recibe el grupo que obtiene resultados se aplica al grupo que no obtiene resultados. No es hasta que la profesora reconoce que ese grupo tiene características propias ("niveles, un piso propio") que brinda una respuesta pertinente a sus necesidades y características. Al reconocer las diferencias, la profesora conforma un nuevo grupo a través de la expresión "de ahi” en la cual se produce la noción de "trabajar en conjunto" fundada en una relación entre profesora y estudiantes, en la que el reconocimiento de las diferencias constituye un facilitador de los procesos de inclusión educativa, brindando a cada uno lo que requiere y asumiendo la improcedencia de asignar lo mismo para todos.

Esta cita ilustra de qué manera la evaluación se posiciona como un mecanismo de control que determina la práctica, desestabiliza y provee incertidumbre a la tarea docente toda vez que al entender la evaluación de resultados como criterio de calidad externo, no es posible para la profesora ejercer su autonomía profesional, sobre la base del análisis contextual y de las características de los y las estudiantes. Pareciera ser que la presencia de "ellos" en ese contexto, vulnera el valor de la igualdad 
produciendo miedo e inseguridad. La necesidad de evaluar a todos los estudiantes por igual y con la finalidad de evidenciar resultados (Stobart, 2008) actúa como barrera en un proceso educativo que demanda diversificación. A diferencia de las citas anteriores la profesora transita desde el miedo, como forma de menosprecio, hacia una experiencia de reconocimiento en la que interactúa con los estudiantes desde la esfera afectiva (Honneth, 2009), lo que le permite responder a sus individualidades.

$\mathrm{Al}$ abandonar las creencias respecto del valor de la igualdad como principio de estandarización y homogeneización del proceso evaluativo, la profesora se abre a una experiencia intersubjetiva (Honneth,2009) a través de la cual logra reconocer en los estudiantes su capacidad de aprender de modos diferentes a los de la mayoría. Promueve por tanto un nuevo valor en su acto educativo que es el respeto por las diferentes formas de aprendizaje y desarrollo humano. Al reconocer las diferencias de aprendizaje de los estudiantes, emerge la noción de un conjunto de estudiantes diversos, que elimina la categorización como subgrupo. Es importante analizar cómo el tipo de relación que se establece entre profesores y estudiantes, está determinada por las percepciones, las emociones y los valores, ya que en un primer momento la profesora establece un vínculo basado en el miedo hacia las diferencias, producto de una expectativa respecto del valor de la igualdad. En cambio, cuando surge el reconocimiento, la profesora logra apreciar el valor de las diferencias que resulta en una comunidad unificada y un trabajo conjunto.

La cita 4 ilustra cómo las intenciones de promover prácticas inclusivas se encuentran permeadas por la homogeneización y la patologización de los y las estudiantes, resultando finalmente en un doble discurso que termina por justificar la segregación.

Cita 4 - Profesora 10: "Yo sí trabajo en pos de la inclusión, y todo eso. Es increíble porque casi siempre me tocan todos los casos más difíciles a mí, es impresionante. Este año por ejemplo, yo tengo niñas con discapacidad cognitiva leve, niñas limítrofes, tengo niñas con hiperactividad a nivel impresionante, de esa que está parada al lado tuyo cada dos minutos, porque todavía no viene la hora del neurólogo y va a venir hasta mayo, entonces tengo marzo, abril y mayo sin darle el medicamento, entonces está todo el rato ahî".

La cita 4 inicia, con un acto de habla declarativo, afirmando un acuerdo y compromiso con la inclusión ("yo sí trabajo en pos de la inclusión”). La expresión " $y$ todo eso", podría estar dando cuenta de dos fenómenos, el primero que se observa como algo ajeno y lejano producto del pronombre "eso", o bien falta de información suficiente sobre la materia para expresar conceptualmente lo que implica ese "todo". En ambos casos, y aun cuando su declaración inicial es enfática en adscribir a la inclusión, la profesora toma distancia de la educación inclusiva, por falta de conocimiento o de sentirse parte activa de ella.

Posteriormente caracteriza a sus estudiantes usando los calificativos "increible" "impresionante" y "casos más difíciles", develando algo fuera de lo común o extraño. No se revela en su discurso un valor positivo respecto de estas diferencias, las que comienzan a ser enumeradas con terminología médica como "discapacidad cognitiva leve", "limitrofes", conceptos que podrían dar cuenta de cómo las caracte- 
rísticas de las estudiantes exceden sus capacidades como docente. Ella reconoce una estudiante que "está parada al lado tuyo cada dos minutos", y sobre la cual se extiende en su argumento recurriendo al discurso médico (Peña, 2013) como las categorías diagnósticas ("hiperactividad a nivel impresionante") la necesidad de ser atendida ("todavia no viene la hora del neurólogo") y los fármacos ("tengo marzo, abril y mayo sin darle el medicamento") como forma de explicar sus características individuales. A través de la partícula cohesiva "entonces", justifica la necesidad de responder a estas características médicas porque mientras no suceda, la niña "está todo el rato ahi”.

El reemplazo del discurso pedagógico por uno médico, resulta útil para argumentar que el problema se encuentra en la niña por alejarse de la normalidad y por tanto deriva la responsabilidad educativa en otros profesionales desconociendo así las barreras educativas y sociales que determinan la participación y progreso de la estudiante (Boggino y Boggino, 2013). Todo lo anterior, pese a declarar que ella sí trabaja "en pos de la inclusión". En este sentido, el discurso médico viene a complementar la tensión entre los valores de la igualdad y del reconocimiento por las diferencias, toda vez que desde la medicina parece ser posible homogeneizar a las estudiantes y concebir sus diferencias (ritmos, identidades, preferencias) como aspectos patológicos factibles de ser recuperados conforme a la normalidad.

En la cita 5, el problema de la evaluación encuentra una estrategia de solución fundada en la distribución de los recursos. La cita trata sobre una profesora hablando sobre un estudiante con Síndrome de Down.

Cita 5 - Profesora 28: "Si [él] estuviera en una escuela especial, estaría con otros niños, similar a él, entonces estábamos hablando que se están entregando por todos lados recursos, entonces de repente sería bueno focalizar un recurso en un solo lugar".

En su discurso la profesora sitúa el valor de la igualdad al plantear que un estudiante pudiera estar "con otros niños, similar a él”. En ese sentido, la profesora propone como solución al problema educativo de la diversidad, el agrupar a los estudiantes de acuerdo con sus características identitarias para así poder focalizar los recursos.

Fraser y Honneth (2003) plantean dos opciones para dar respuesta a las diferencias de la población. El primero es el reconocimiento a través del cual se asume la diversidad social trabajándose sobre ella, mientras que la redistribución, implica una asignación de recursos diferente. El argumento de la profesora devela la redistribución de los recursos pedagógicos, materiales y humanos, agrupando a los estudiantes según sus características. Se niega por tanto la diversidad de estudiantes y se intenta de esta forma, generar dos grupos homogéneos para poder asignarles apropiadamente los recursos.

En este sentido cuando el problema es abordado primero desde la redistribución de los recursos, se deja de considerar la diversidad del grupo en cuestión (Fraser y Honneth, 2003) justificándose la segregación y exclusión. El abordaje pedagógico que implica la diversidad de estudiantes, podría resolverse desde el reconocimiento si se analizaran primero las características diversas del grupo, se determinaran los recursos que se requieren para atender a esa diversidad, y se realizara 
luego, la flexibilidad necesaria (Rose y Gavel, 2010). Sin embargo, la pretensión de igualdad parece operar antes que la de reconocimiento y la estrategia que aparece es la de redistribuir.

En la cita 6, el profesor entrevistado refuerza la idea de la homogeneización de los estudiantes como un valor inherente al sistema educativo.

Cita 6 - Profesor 21: "Por lo menos yo no percibo que sea como, excluyente al resto, sino que, incluso hay niños que son del PIE que son mucho más habilidosos que el grupo, nor... digamos normal entre comillas, así que, creo que se integró bien".

El profesor utiliza nuevamente la estrategia de conformar grupos de estudiantes a través del lenguaje, diferenciando a los niños "que son del PIE" de los otros niños y niñas. Permanece así, la idea de dentro y fuera que plantean Boggino y Boggino (2013). Utilizando mitigadores como "digamos", "normal entre comillas" y un "nor..." que no alcanza a finalizar, caracteriza ambos grupos: uno de niños y niñas normales y otro de niños y niñas "que son del PIE". Estos mitigadores, evidencian que el profesor tiene consciencia de que su caracterización no es aceptada socialmente, sin embargo persiste en su idea puesto que la conformación de grupos es la estrategia discursiva que le permitirá justificar su argumento. Habiendo entonces, realizado esta diferencia entre estudiantes, el profesor justifica la buena integración cuando el grupo que es "del PIE" (el grupo distinto del normal), logra ser "mucho más habilidoso que el grupo nor... digamos normal entre comillas". En este punto, el profesor utiliza la conjunción "incluso", la cual podría dar cuenta de bajas expectativas respecto de aquel grupo que logró igualarse o superar al grupo normal.

Aunque se establecen diferencias entre estudiantes en grupos, el profesor señala que no percibe exclusión. Este fenómeno bien puede estar determinado por la atribución de un reconocimiento normativo (Honneth, 2009), que reconoce a los estudiantes como sujetos con derecho a la educación, pero desconoce el reconocimiento ético de sus características individuales. Para el profesor, el hecho de que los estudiantes estén en la misma sala, ya es razón suficiente para dar por garantizada la inclusión y por rechazada la exclusión.

Sin embargo bajo esta lógica homogeneizadora, los estudiantes no encontrarán reconocimiento sino en la medida en que se asemejen al grupo de estudiantes considerados normales. Este enunciado, en el cual el profesor describe un proceso de integración y no de inclusión, la experiencia es exitosa en la medida que un grupo se asemeje al otro, permitiendo hacer dos inferencias respecto de la accesibilidad que brinda el establecimiento (Boggino y Boggino, 2013). La primera es que la estrategia de respuesta a la diversidad de la escuela es justamente reducir la diversidad, por tanto los esfuerzos estarían concentrados en homogeneizar a los grupos. La segunda inferencia es que la responsabilidad de adaptarse (integrarse) al establecimiento, recae en los sujetos y no en las posibilidades de accesibilidad que ofrece la escuela. Asemejarse al grupo normal, en este sentido, significa aparentar normalidad respondiendo nuevamente al valor de la igualdad entendido en este contexto como, estudiantes con conductas, niveles de aprendizaje, ritmos y formas de aprendizaje similares. 
Finalmente, en la cita 7 la profesora refiere al trabajo colaborativo con educadoras diferenciales, en tanto estrategia para la educación inclusiva en el aula.

Cita 7 - Profesora 8: "La profesional tiene que atender a todas las niñas no solamente a la niña que está más descendida, obviamente también la atiende, pero si atiende, porque en los cursos son 45 , ¿Qué pasa con la que está más descendida? Que pasó en unos segundos y siguió donde la otra que está diciendo tía necesito ayuda, que no está tan descendida. Entonces ahí no sé, digamos, no hay un aprendizaje como tan significativo, si esa misma alumna se saca de la sala se trabaja con estos niños, que está más descendido, los resultados son otros, créame, porque yo he visto las dos partes, la escuela antigua y la nueva escuela".

Esta cita resulta útil para sintetizar la tesis de que el modelo educativo tradicional necesita homogeneizar a los estudiantes para poder sostenerse. Utilizando el verbo deóntico "tiene que", deja entrever que la educadora diferencial tiene una función obligada que es "atender a todas las niñas", la cual contrasta con la pregunta “¿Qué pasa con la que está más descendida?". En este contexto, "la más descendida" constituye un sujeto colectivo que representa a toda estudiante que no avanza igual que sus pares y asimismo opera como un eufemismo para enmascarar las bajas expectativas respecto de esa o esas estudiantes. El verbo de identidad "estâ", atribuye el rasgo de descendido a la estudiante y no al contexto educativo. En este sentido, el foco deja de estar en la estudiante y pasa a estar en la condición de descendida (característica indeseada). La niña descendida, entonces, no recibe la atención necesaria porque está la "que no está tan descendida" resultando nuevamente la conformación de grupos una estrategia discursiva útil para justificar la anulación de las diferencias.

$\mathrm{Al}$ respecto, el discurso devela su intención final, a través de algunos mitigadores "ahi no sé", "digamos" y recurriendo a un concepto potente para la pedagogía como es la necesidad del aprendizaje significativo, se plantea directamente la segregación como estrategia: "si esa misma alumna se saca de la sala se trabaja con estos niños". Las diferencias no solo se eliminan en el plano simbólico, sino que explícitamente deben eliminarse de los planos físicos y educativos. Para justificar esta exclusión, recurre a una experiencia personal incuestionable respecto de los resultados, la que expone a través de un acto de habla apelativo "créame, porque yo he visto las dos partes".

En este sentido, no se formulan cuestionamientos respecto de la organización del espacio pedagógico, de la implementación de estrategias de diversificación de enseñanza, del número de estudiantes por sala, del rol de la educadora diferencial, del trabajo colaborativo o la coenseñanza, elementos contextuales ausentes del discurso. Denota entonces, el discurso docente, una falta de formación respecto de la educación inclusiva y de los instrumentos jurídicos implementados para normarla.

\section{DISCUSIÓN}

El análisis crítico del discurso realizado al relato de las experiencias de inclusión educativa de profesores en ejercicio, devela estrategias de agrupación de estudiantes, el uso de verbos deónticos y mitigadores, las cuales contribuyen a justificar experiencias de menosprecio hacia las diferencias de los y las estudiantes. 
El principio de igualdad de oportunidades que sustenta el discurso docente, les permite justificar la conformación de grupos de estudiantes en base a criterios evaluativos como la estandarización de resultados y estrategias de aprendizaje. La respuesta que se brinda a las diferentes formas de aprender de los estudiantes es una obligación legal que termina asimilándose a la de aquellos que tradicionalmente han estado en la educación (Boggino y Boggino, 2013). En ese sentido, educar en diversidad resulta un proceso que da respuesta a un reconocimiento normativo más que a un reconocimiento ético, motivado más por las leyes implementadas y menos por un compromiso frente a la exclusión de determinadas personas producto de sus características individuales (Honneth, 2009).

La fuerte presencia del valor de la igualdad (Dubet, 2014) hace tensionar la idea de que la segregación es indeseada, con la presencia efectiva de prácticas o ideas segregadoras. En ese sentido cuando los estudiantes no son iguales entre sí, aparece la conformación de grupos (según capacidad, nivel de logro, conducta). Las concepciones de los profesores respecto del principio de igualdad focalizan el fenómeno educativo en las características de los estudiantes y no en los contextos pedagógicos, justificando la agrupación de estudiantes por sus características y por tanto la segregación. Al justificarse la segregación se anula la posibilidad de diversificar las formas de participación y los progresos académicos (Boggino y Boggino, 2013; Booth y Ainscow, 2015).

La evaluación aparece como el elemento normativo que sobre criterios de calidad externos, obliga a los docentes a tomar decisiones (Stobart, 2008) fundamentadas en el principio de igualdad entendido como estandarización, homogeneización y finalmente normalización. Es desde esta concepción que aparecen experiencias de menosprecio como la segregación y la negación de las diferencias de los y las estudiantes. Al operar de esta manera, la evaluación se enfoca de manera clínica (Peña, 2013) y no pedagógica, buscando explicar las dificultades en la biología del estudiante más que en los contextos de aprendizaje. Así entendida, la evaluación cuestiona el cuerpo físico y las estructuras psicológicas, que son parte constitutiva de la identidad de los estudiantes (Lafferriere, 2018) al tiempo que permanece silenciosa respecto de las formas de enseñanza, los patrones de relación social y las propias creencias docentes.

Las concepciones docentes respecto de las dificultades de aprendizaje operan desde una visión estática, inherente al estudiante, más que desde una visión situacional, dependientes del ambiente (Echeita et al., 2013) donde la evaluación, a través del diagnóstico clínico, niega las múltiples condiciones humanas, reduciéndolas a patologías que deben ser corregidas.

La evaluación se constituye como una barrera para un diseño educativo respetuoso de las características individuales de los estudiantes (Muñoz, López y Assaél, 2015), y por tanto, sus resultados solo son utilizables en favor de la homogeneización. En este proceso existe un ideal de estudiante que determina una forma de diseño educativo (Rose y Gravell, 2010) y que opera desde un sistema de recompensa y castigo según los resultados obtenidos en función de ese ideal.

Este mecanismo asociado a la evaluación se enmarca en un contexto político chileno basado en la competencia y la calidad entendida como resultados de apren- 
dizaje (Bellei, 2015; Falabella y De la Vega, 2016). La Ley General de Educación n. 20.370 define la calidad educativa como una serie de objetivos y estándares que deben ser alcanzados por los y las estudiantes. Asimismo, crea una Agencia de la Calidad de la Educación encargada de la evaluación con base a estándares indicativos. Este tipo de políticas, coexisten con aquellas orientadas a eliminar la segregación y promover el acceso de nuevos colectivos como la Ley de Inclusión Escolar (Chile, 2015b) o la Ley Antidiscriminación (Chile, 2012).

Las contradicciones políticas han obligado a los docentes a armonizar ambos discursos ante la imposibilidad de adoptar uno y rechazar otro, en tanto constituyen obligaciones simultáneas. La estrategia utilizada frente a este fenómeno es reemplazar los principios de base presentes en cada política para alcanzar la coherencia (Jobert, 2008; Retamozo y Stoessel, 2014). Así la idea de la segregación fue reemplazada por el principio de igualdad, el cual sin embargo termina justificando el mismo proceso de exclusión y marginación. $\mathrm{Al}$ aplicarse el principio de igualdad sobre el reconocimiento normativo, entonces todos y todas tienen el mismo derecho a la educación. Sin embargo, al aplicar la igualdad sobre el reconocimiento ético, entonces todos y todas deben aprender lo mismo y de la misma manera. De esta forma se argumentan acciones como agrupar o clasificar estudiantes similares, justificándose así nuevamente la segregación.

Un problema de la educación inclusiva en Chile, es que el sistema educativo ya ha reconocido características y habilidades (Boggino y Boggino, 2013; Santos, 2013) que permiten conformar grupos de estudiantes. Esto queda en evidencia en los estándares evaluativos de calidad de la Ley General de Educación y en las clasificaciones diagnósticas del decreto n. 170 (Peña, 2013). Por tanto se puede afirmar que en Chile, los mecanismos legales de acceso que dotan a los individuos de reconocimiento normativo, no contemplan mecanismos para dotarles de reconocimiento ético.

Esta debilidad del sistema legal chileno, promueve que aquellos grupos, tradicionalmente excluidos que ahora acceden al sistema educativo, no tengan más alternativa que asimilar sus características a las de aquellos que tradicionalmente han formado parte de dicho sistema. Las estrategias de respuesta a la diversidad implementadas no se justifican para dotarles de reconocimiento ético (que aprendan conforme a sus características por ejemplo), sino que se justifican para que el grupo que tradicionalmente ha estado en el sistema, pueda continuar aprendiendo a pesar de la presencia de estos nuevos grupos.

De esta forma, el menosprecio no se expresa solo a nivel simbólico, considerando indeseables los modos alternos de aprendizaje, sino que trasciende al nivel físico buscando estrategias para eliminar, segregar o excluir dichas diferencias de los espacios comunes. Esta experiencia de menosprecio termina en la cosificación (Honneth, 2009) del estudiante, quien abandona su condición de sujeto de derecho y se transforma primero en un obstáculo para los intereses de grupos mayoritarios y luego en un objeto de rehabilitación y asimilación de características deseadas.

Para superar estas experiencias de menosprecio es necesario habilitar más espacios de participación en el debate en condiciones horizontales (Casuso, 2018; Habermas, 1981; Honneth, 2014). Sin embargo, al carecer los estudiantes de reconocimiento ético, su participación podría ser limitada o casi inexistente (Reyes, 2019). 
Este análisis no ha pretendido ser una respuesta definitiva a la problemática de la segregación y la educación inclusiva. Desde un contexto local se ha propuesto ilustrar cómo el discurso docente constituye un reflejo de políticas educativas contradictorias y de la tensión que existe entre ellas.

La falta de reconocimiento de la institución educativa hacia la diversidad de formas de desarrollo, aprendizaje, participación y progreso podría estar operando como un primer factor que permea todas las intenciones materiales de dar una respuesta educativa (formación docente, gestión, distribución de recursos, programas, políticas, entre otros).

En ese sentido se podría pensar que, mientras las políticas y las instituciones educativas continúen sustentadas en el principio de igualdad respecto de una sola forma de aprender; mientras se mantenga la idea de que existen grupos buenos y grupos malos de estudiantes; $y$ mientras persista la concepción de aprendizaje como resultado medible y comparable entre individuos, los esfuerzos por alcanzar una reconocimiento ético de la diversidad de formas de ser y aprender, quedarán relegados a estrategias que justificarán la segregación y mantendrán inamovible el sistema educativo.

Este fenómeno podría abrir el debate hacia una nueva forma de menosprecio que no tiene relación con la pobreza, el género o la discapacidad, sino que lo que tanto políticas como instituciones educativas en específico menosprecian, son las diferentes formas de ser de los y las estudiantes. De esta manera, no es posible que los grupos minoritarios o tradicionalmente excluidos puedan aprender en una institución que no solo no está pensada para ellos y ellas, sino que además no quiere ni reconoce las múltiples formas particulares de acceder, procesar y construir el conocimiento.

\section{REFERENCIAS}

ALBORNO, N. E.; GAAD, E. “Index for Inclusion”: a framework for school review in the United Arab Emirates. British Journal of Special Education, Manchester, v. 41, n. 3, p. 231-248, sept. 2014. https://doi.org/10.1111/1467-8578.12073

BELLEI, C. El gran experimento. Mercado y privatización de la educación Chilena. 1. ed. Santiago: LOM, 2015.

BERNABÉ, M.; ALONSO, V.; BERMELL, M. Evolución de la terminología relacionada con la atención a la diversidad a través de las diferentes reformas legislativas en España. Revista Ibero-Americana de Educação, España, v. 70, n. 1, p. 79-96, 2016. https://doi.org/10.35362/rie70174

BOGGINO, N.; BOGGINO, P. Pensar una escuela accesible para todos. 1. ed. Rosario: Homosapiens, 2013.

BOOTH, T.; AINSCOW, M. Guía para la educación inclusiva. Desarrollando el aprendizaje y la participación en los centros escolares. 2. ed. Madrid: OEI, 2015.

CAMERON, D.; PANOVIC, I. Working with writtern discourse. 1. ed. London: Sage, 2014.

CASUSO, G. Disonancia y crítica social. Apuntes sobre la relación entre el poder y la exclusión. In: CASUSO, G.; SERRANO, J. (ed.). Las armas de la crítica. Lima: Anthropos, 2018. p. 41-67. 
CHILE. Decreto n. 170. Decreto con toma de razón n. 170. Fija normas para determinar los alumnos con necesidades educativas especiales que serán beneficiarios de las subvenciones para educación especial. Santiago: Ministerio de Educación, 14 mayo 2009a.

CHILE. Ley n. 20.370, 17 de agosto de 2009. Establece la Ley General de Educación. Diario Oficial de la República, Santiago, 12 sept. $2009 \mathrm{~b}$.

CHILE. Ley n. 20.422, 3 febrero de 2010. Establece normas sobre igualdad de oportunidades e inclusión social de personas con discapacidad. Diario Oficial de la República, Santiago, 10 dic. 2010.

CHILE. Ley n. 20.609, 12 julio de 2012. Establece medidas contra la discriminación. Diario Oficial de la República, Santiago, 24 jul. 2012.

CHILE. Decreto n. 83, 30 de enero de 2015. Aprueba criterios y orientaciones de adecuación curricular para estudiantes con necesidades educativas especiales de educación parvularia y educación básica. Diario Oficial de la República, Santiago, 5 feb. 2015a.

CHILE. Ley n. 20.845, 29 de mayo de 2015. De inclusión escolar que regula la admisión de los y las estudiantes, elimina el financiamiento compartido y prohíbe el lucro en establecimientos educacionales que reciben aportes del Estado. Diario Oficial de la República, Santiago, 8 jun. 2015b.

DUBET, F. Repensar la justicia social. Contra el mito de la igualdad de oportunidades. 3. ed. Buenos Aires: Siglo XXI, 2014.

ECHEITA, G.; NAVARRO, D. Educación inclusiva y desarrollo sostenible. Una llamada urgente a pensarlas juntas. Edetania-Estudiosy Propuestas Socio-Educativas, España,v. 46, n. 2, p. 141-161, 2015.

ECHEITA, G.; SIMÓN, C.; LÓPEZ,M.; URBINA, C. Educación inclusiva. Sistemas de referencia, coordenadas y vórtices de un proceso dilemático. In: VERDUGO, M. Á.; SHALOCK, R. (coords.). Discapacidad e inclusión. Manual para la Docencia. Salamanca: Amaru, 2013. p. 329-357.

ENGELBRECHT, P.; SAVOLAINEN, H.; NEL, M.; KOSKELA, T.; OKKOLIN, M.-A. Making meaning of inclusive education: classroom practices in Finnish and South African classroom. Compare: a Journal of Comparative and International Education, v. 47, n. 5, p. 684-702, 2017. http://dx.doi.org/10.1080/03057925.2016 .1266927

FAIRCLOUGH, N. El análisis crítico del discurso y la mercantilización del discurso público: las universidades. Discurso \& Sociedad, Barcelona, v. 2, n. 1, p. 170-185, 2008. FALABELLA, A.; DE LA VEGA, L. F. Políticas de responsabilización por desempeño escolar: un debate a partir de la literatura internacional y el caso chileno. Estudios Pedagógicos, Valdivia, v. 42, n. 2, p. 395-413, 2016. http://dx.doi.org/10.4067/S071807052016000200023

FRASER, N.; HONNETH, A. ¿Redistribución o reconocimiento? Un debate político-filosófico. 1. ed. Madrid: Morata, 2003.

HABERMAS, J. Teoría de la acción comunicativa. 1. ed. Madrid: Taurus, 1981. 
HABERMAS, J. Aclaraciones a la ética del discurso. 1. ed. Madrid: Trotta, 1991. HATTIE, J. Aprendizaje visible para profesores. Maximizando el impacto en el aprendizaje. 3. ed. España: Paraninfo, 2017.

HOBBES, T. Leviatán o la materia forma y poder de un estado eclesiástico y civil. 3. ed. Madrid: Fondo de Cultura Económica, 1651.

HONNETH, A. La lucha por el reconocimiento: por una gramática moral de los conflictos sociales. 1. ed. Barcelona: Crítica, 1992.

HONNETH, A. Crítica del poder. Fases en la reflexión de una teoría crítica de la sociedad. 1. ed. Madrid: Machado, 2009.

HONNETH, A. El derecho de la libertad. Esbozo de una eticidad democrática. 1. ed. Buenos Aires: Kats, 2014.

JOBERT, B. Estado, sociedad, políticas públicas. Santiago: Universitaria, 2008.

LAFFERRIERE, J. N. ¿Materia disponible o realidad personal? El cuerpo humano, las biotecnologías y las exigencias jurídicas de la dignidad. Sociología y Tecnociencia, España, v. 8, n. 1, p. 60-84, 2018. https://doi.org/10.24197/st.1.2018.60-84

LÓPEZ, V.; JULIO, C.; PÉREZ, M. V.; MORALES, M.; ROJAS, C. Barreras culturales para la inclusión: políticas y prácticas de integración en Chile. Revista de Educación, España, v. 363, n. 1, p. 256-281, 2014. http://dx.doi.org/10-4438/1988592X-RE-2012-363-180

MACKEY, M. Inclusive education in the United States: Middle School General Education Teacher's Approaches to Inclusion. International Journal of Instruction, Switzerland, v. 7, n. 2, p. 5-20, 2014.

MELlADO, M. E.; CHAUCANO, J. C.; HUECHE, M.; ARAVENA, O. Percepciones sobre la educación inclusiva del profesorado de una escuela con Programa de Integración Escolar. Revista Educación, España, v. 41, n. 1, p. 1-14, 2017. http:// dx.doi.org/10.15517/REVEDU.V41I1.21597

MUÑOZ, M. L.; LÓPEZ, M.; ASSAÉL, J. Concepciones docentes para responder a la diversidad: ¿Barreras o recursos para la inclusión educativa? Psicoperspectivas. Individuo y Sociedad, Valparíso, v. 14, n. 3, p. 68-79, 2015.http://dx.doi.org/10.5027/ psicoperspectivas-Vol14-Issue3-fulltext-646

OECD - Organisation for Economic Co-operation and Development. Evaluaciones de Políticas Nacionales de Educación. Educación en Chile. [S.l.]: OECD, 2017. Disponible en: http://archivos.agenciaeducacion.cl/Educacion_en_Chile_OCDE_ Nov2017.pdf. Acceso en: 30 enero 2018.

ONU - Naciones Unidas. Informe nacional presentado con arreglo al párrafo 5 del anexo de la resolución del Consejo de Derechos Humanos. Chile: Naciones Unidas, 2019. Disponible en: https://documents-dds-ny.un.org/doc/UNDOC/GEN/ G18/333/78/PDF/G1833378.pdf?OpenElement. Acceso en: 1 feb. 2019.

PEÑA, M. Análisis crítico de discurso del decreto 170 de subvención diferenciada para necesidades educativas especiales: el diagnóstico como herramienta de gestión. Psicoperspectivas. Individuo y Sociedad, Valparíso, v. 12, n. 2, p. 93-103, 2013. http:// dx.doi.org/10.5027/PSICOPERSPECTIVAS-VOL12-ISSUE2-FULLTEXT-252 
RAWLS, J. Teoría de la justicia. 2. ed. México: Fondo de Cultura Económica, 1971. RETAMOZO, M.; STOESSEL, S. El concepto de antagonismo en la teoría política contemporánea. Estudios Políticos, Medellín, n. 44, p. 13-34, 2014.

REYES, L. Barreras y facilitadores en la inclusión de estudiantes sordos en un liceo técnico profesional de la comuna de Nuñoa. 2019. Tesis (Magíster en Educación) Universidad de Chile, Santiago, 2019.

ROSE, D. H.; GRAVEL, J. W. Universal design for learning. In: PETERSON, P.; BAKER, E.; MCGRAW, B. (eds.). International enciclopedia of education. Oxford: Elsevier, 2010. p. 119-124.

SALAS, R. Teorías contemporáneas del reconocimiento. Atenea, Concepción, n. 514, p. 79-93, 2017. http://dx.doi.org/10.4067/S0718-04622016000200079

SANTOS, B. Desvolonizar el saber, reinventar el poder. 1. ed. Santiago: LOM, 2013. SENADIS. II Estudio de la Discapacidad. Un nuevo enfoque para la inclusión 2015. Santiago: SENADIS, Ministerio de Desarrollo Social, 2015. Disponible en: https:// www.senadis.gob.cl/descarga/i/3959. Acceso en: 30 enero 2016.

STOBART, G. Testing times. The uses and abuses of assessment. 1. ed. New York: Routledge, 2008.

UNESCO - United Nations Educational, Scientific and Cultural Organization. Education for All 2000-2015: achievements and challenges. Paris: UNESCO, 2015. Disponible en: http://unesdoc.unesco.org/images/0023/002322/232205e.pdf. Acceso en: 15 abr. 2016.

UNESCO - United Nations Educational, Scientific and Cultural Organization. Desglosar el objetivo de desarrollo sostenible 4 educación 2030. 2017. Disponible en: http://unesdoc.unesco.org/images/0024/002463/246300S.pdf. Acceso en: 14 jul. 2018. VAN DIJK, T. Discurso y poder. 1. ed. Barcelona: Gedisa, 2009.

VILLALOBOS, M. Ciencia social como crítica social. La revolución epistemológica de Karl Marx. 1. ed. Santiago: LOM, 2010.

\section{SOBRE LOS AUTORES}

Jorge Eduardo Alfaro es doctor en ciencias de la educación por la Universidad de La Frontera (Chile).

E-mail: jorgeeduardoau@gmail.com

Valeria Herrera Fernández es doctora en psicología por la Universidad Complutense de Madrid (España). Profesora de la Universidad de Chile (Chile). E-mail:valherrera@u.uchile.cl 